\title{
Erratum to: Evaluation of Tree Forwarding by Farm Tractor in Patch Cutting of Poplar Plantations in Northern Iran
}

\author{
Seyed Rostam Mousavi Mirkala ${ }^{1}$. \\ Mehrdad Nikooy ${ }^{2}$
}

\section{Erratum to: Small-scale Forestry (2014) 13:527-540 DOI 10.1007/s11842-014-9269-3}

For Urmia University to recognize this publication as Seyed Rostam Moustavi's, it is important that his affiliation is corrected from University of Urmia to Urmia University.

\section{Seyed Rostam Mousavi Mirkala}

rostammousavi@yahoo.com; r.mousavi@urmia.ac.ir

Mehrdad Nikooy

mehrdad.nikooy@gmail.com

1 Faculty of Natural Resources, Urmia University, Urmia, Iran

2 Department of Forestry, University of Guilan, Somea Sara, Guilan, Iran 